Bull. Mater. Sci., Vol. 22, No. 3, May 1999, pp. 227-231. (C) Indian Academy of Sciences.

\title{
Direct observation of the interaction between vortices and dislocations in superconducting crystals by a cryo-Lorentz EM (interaction between vortices and dislocations)
}

\author{
SHIGEO HORIUCHI*, MARCO CANTONI, MASAYA UCHIDA, TADAMASA TSURUTA and \\ YOSHIO MATSUI
}

National Institute for Research in Inorganic Materials, Namiki 1-1, Tsukuba, Ibaraki 305-0044, Japan

\begin{abstract}
Quantized magnetic flux lines (vortices) in a $\mathrm{Nb}$ foil were directly observed in different magnetic fields up to $200 \mathrm{G}$ by a cryo-Lorentz electron microscope. The interaction of vortices with dislocations in the specimen was examined and clarified; edge-on dislocations weakly pin individual vortices at magnetic fields below $100 \mathrm{G}$. In higher magnetic fields the formation of a regular hexagonal vortex lattice started preferentially at in-plane dislocations. At $200 \mathrm{G}$ the Abrikosov vortex lattice was formed with small domains, whose centre included the dislocations, showing their important role on the formation of the vortex lattice. For a NbTi foil no clear image of vortices could be seen, because the surface was rough due to the formation of fine grains and precipitates.
\end{abstract}

Keywords. Nb; interaction of vortices with dislocations; cryo-Lorentz electron microscope; vortex pinning; Abrikosov lattice; NbTi.

\section{Introduction}

In order to use high- $T_{\mathrm{c}}$ superconductors (HTSCs) practically as device materials, magnet materials, etc high values not only of the transition temperature $T_{\mathrm{c}}$ but also of the critical current density $J_{c}$ under external magnetic fields are necessary. The latter depends on how superconducting grains are linked to each other and what their orientation is. The most effective factor for type 2 superconductors is that vortices can be pinned, because the superconducting state gets deteriorated when quantized magnetic flux lines (vortices) move.

The strength for pinning vortices is not an inherent nature of substances but determined by the fine structures in materials. It is therefore important to clarify the mechanism of vortex pinning in order to attain the high $J_{c}$ values. It has however been still unclear what the actually effective pinning sites are and how they achieve pinning. By means of conventional transmission electron microscopy we have tried to identify possible pinning sites in some $\mathrm{Bi}-, \mathrm{Tl}$ - and Y-based HTSCs (Wu et al 1991; Jeong et al 1996; Cantoni et al 1997).

Observation of vortices have been done by some methods. For example, the technique of decorating vortices in superconductors by ferromagnetic particles is well established and called the Bitter method. The technique has been extended and combined with the thin film observation by a transmission electron microscopy (Herring 1974). On the other hand, cryo-Lorentz electron

*Author for correspondence microscopy (CLEM) offers a powerful tool to directly observe vortices in $\mathrm{Nb}$ (Harada et al 1992) and in Bi-based HTSCs (Harada et al 1993). Their dynamic behaviour was examined in externally applied magnetic fields below $150 \mathrm{G}$. Furthermore, the interaction of vortices with artificial hole-like defects has been clarified (Harada et al 1996).

In order to clarify the interaction between vortices and actual lattice defects in superconductors, we have recently developed a new type of CLEM with a $300 \mathrm{kV}$ field emission gun (Hitachi HF-3000L) which enables us to apply magnetic fields up to $300 \mathrm{G}$ unidirectionally (Cantoni et al 1998; Horiuchi et al 1998). Specimens can be cooled down to $5 \mathrm{~K}$. The present study has been focused on elucidating the relationship between the vortices or the vortex lattice and dislocations in $\mathrm{Nb}$.

\section{Experimental}

Thin films of high purity $\mathrm{Nb}\left(T_{\mathrm{c}}=9.2 \mathrm{~K}\right)$ were prepared by chemical- or electro-polishing and then mounted in the side-entry-type specimen holder. The specimen was tilted relative to the electron beam by $45^{\circ}$ and cooled to $5 \mathrm{~K}$ during the observation. Images were taken at a direct magnification of $\times 1000$ with a defocus of about $10 \mathrm{~mm}$. Imaging plates were used to record images in order to shorten the exposure time ( $5 \mathrm{sec}$ compared to about $30 \mathrm{sec}$ on photographic films). The magnetic field was applied perpendicular to the incident electron beam as well as to the tilting axis. 


\section{Results and discussion}

\subsection{Interaction between individual vortices and dislocations in low magnetic fields}

In externally applied fields below $100 \mathrm{G}$ vortices arranged irregularly. Their density depended on the field strength and the specimen thickness. Vortices were pinned individually to weak pinning sites at least in short periods. The minimization of line energy seemed to attract them to thinner parts of the specimen. The distribution and strength of the involved pinning sites were examined as follows: the specimen was cooled below $T_{\mathrm{c}}$ $(5 \mathrm{~K})$ without any magnetic field. On applying a field of $12 \mathrm{G}$ vortices entered the specimen film from the edge. At this moment they felt the repulsion due to the line tension and the attraction to weak pinning sites on their way. Although a major part of vortices penetrates the $\mathrm{Nb}$ film almost instantaneously on applying the field, an equilibrium will be reached after several minutes. During this time vortices can be observed hopping from one pinning site to the other towards thicker regions. Figures $1 \mathrm{a}$ and $\mathrm{b}$ were taken just on applying the field and $6 \mathrm{~min}$ later, respectively.

Figure 1c summarizes the observation; positions of vortices found at the beginning are marked by open circles, while end positions by small dots. Large dots represent strong pinning sites occupied by the same vortices both in figures $1 a$ and $b$. The number's ratio of the large dots to all the spots is about 0.16. The density of the large dots is not uniform but the average one is counted to be $1.7 \times 10^{7} / \mathrm{cm}^{2}$. We may say that positions of large dots are effective pinning sites. A similar result has been reported very recently, in which a rather random distribution of pinning sites in a $\mathrm{Nb}$ foil was observed (Sow et al 1998). The origin of the pinning force at the sites of large dots may be due to dislocations, although the identification of them was difficult in this case, because they lay 'edge-on' and their image contrast is superposed on that of vortices.

In another film we have occasionally found an area, in which the slip trace of dislocations was prominent and possible to identify them. Figure 2 is a bright-field image of such an area. Edge-on dislocations can clearly be seen at their tips, as marked by dark open circles. An enlarged image in the lower right inset clearly shows an edge-on dislocation at the end of a slip trace. Dislocations, which are not related to slip traces, are also seen as marked by white open circles. A CLEM image was taken from the same area with an applied field of $45 \mathrm{G}$ at $5 \mathrm{~K}$. It is partly superimposed in the central upper part of figure 2 to show how vortices occur. Every vortices are plotted by dark dots not only in the area of the CLEM image inserted but also in the area for which the CLEM image is not superposed. By comparing the dark and white open circles with the dark dots we can recognize a relationship between the positions of vortices and dislocations as follows.

The density of vortices varies over the observed area. The vortex density is higher $\left(\sim 5 \times 10^{8} / \mathrm{cm}^{2}\right)$ in the upper centre area, where the specimen is apparently thin, as compared to adjoining areas. The upward bending of the broad thickness contours in the left part of figure 2 indicates a decrease of the thickness towards the central part of the image. The vortices are attracted to thin parts and arrange in a more regular way. On the left-hand side and the right-hand side, on the other hand, the vortex densities are relatively low. In these thick parts
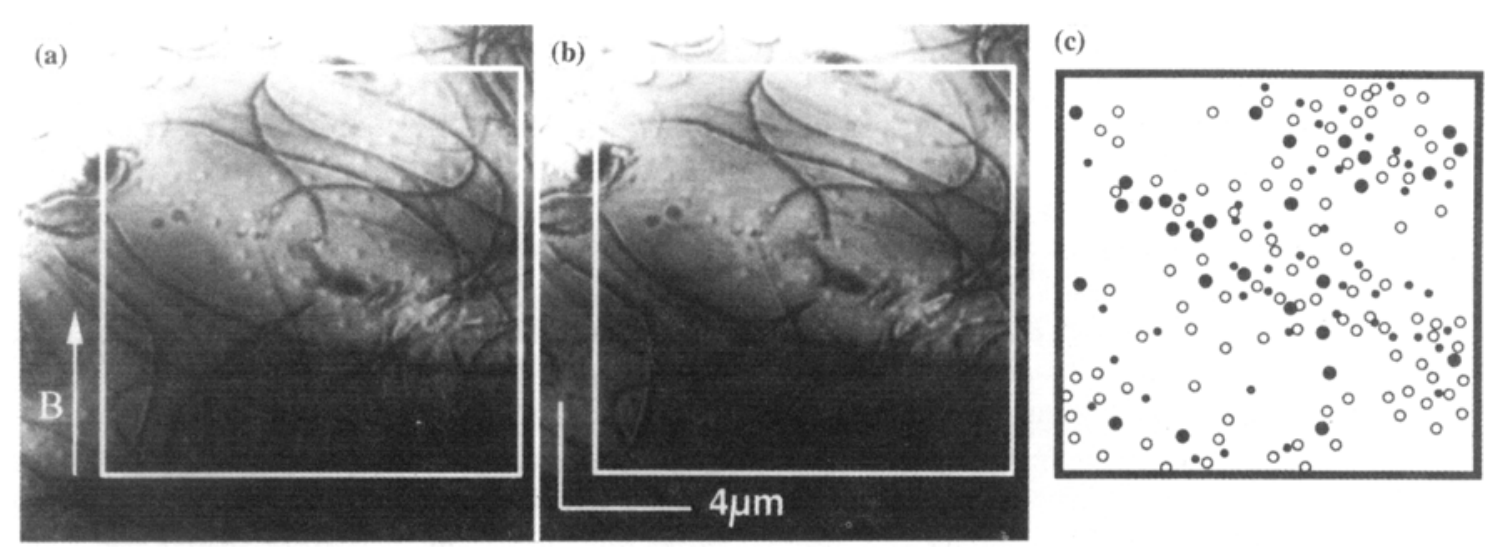

Figure 1. (a) A CLEM image of $\mathrm{Nb}$ taken a few sec after applying an external magnetic field of $12 \mathrm{G}$. Specimen temperature is $5 \mathrm{~K}$. Vortices appear as small globules with a pair of dark and bright contrast. Dark lines are bend contours. The direction of applied magnetic field is shown by B. (b) Same area as (a) but 6 min later. During this time vortices moved into the specimen from the left top to the right bottom towards thicker regions. (c) Vortex positions in marked frames in (a) and (b) are compared. Positions of vortices in (a) are marked by open circles, while those in (b) by small dots. Large dots represent effective pinning sites occupied by vortices common in (a) and (b). 
the vortex positions often coincide with the edge-on dislocations. This observation strongly suggests that vortices are effectively pinned to the dislocations. However, not all edge-on dislocations are able to trap vortices. We may therefore conclude that their pinning potential is rather weak and vortices tend to move to thinner areas. Other weak pinning sites, which affect the arrangement of vortices at low magnetic fields, may be small steps at the specimen surface, relating to the variation of the specimen thickness. A sharp change in thickness near subgrain boundaries has been found to trap vortices on the thinner side during the observation of their dynamic behaviour (Harada et al 1994).

\subsection{Interaction between the Abrikosov vortex lattice and dislocations in high magnetic fields}

In fields of above $100 \mathrm{G}$ the density of vortices increases. The interaction of vortices with each other competes with what was related to individual pinning sites mentioned above. The variation of vortex density is much smaller and they are more uniformly arranged.

At $100 \mathrm{G}$ vortices with increased density are locally ordered forming a lattice with hexagonal symmetry, as shown in figure $3 \mathrm{a}$. Thick curved lines are bend contours. A thin curved line, marked by asterisks, is a dislocation lying in the $\mathrm{Nb}$ film ('in-plane' dislocation). How to discriminate dislocations from bend contours will be mentioned below, relating to figure 4 . It is noted that

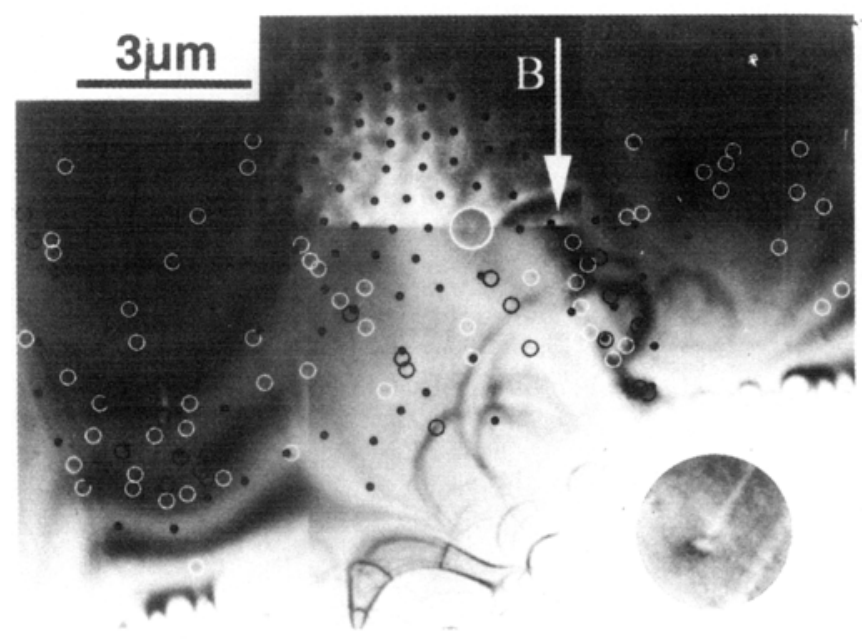

Figure 2. A bright-field TEM image of $\mathrm{Nb}$ taken from an area containing many slip traces and edge-on dislocations at their tips, which are marked by dark open circles. Dislocations, not related to slip traces, are marked by open circles. The inset in the lower right corner is an enlarged image of the area marked by a large circle and clearly shows an edge-on dislocation at the end of a slip trace. The inset in the upper centre part is a superposed CLEM image, which was taken under $45 \mathrm{G}$ at $5 \mathrm{~K}$, indicating the positions of vortices, as marked by dark dots. Effective pinning sites can be read out. the vortices around the dislocation seem to array in order, as schematically depicted in figure $3 \mathrm{~b}$. This means that a vortex lattice forms locally.

It should be mentioned here that the starting sites of such a vortex lattice is not always at dislocations. Lattice irregularities such as surface defects including steps, inclusions and grain boundaries are also possible starting sites. But dislocations seem to play this role most prominently at this stage.

With the increase of the magnetic field the vortex density increased. At $200 \mathrm{G}$ vortices became very dense and formed a rather regular vortex lattice (figure 4). Figure 4a was taken from the same area as figure 3 but with a different direction of applied magnetic field. The vortex lattice is disordered in a region along a downward arrow, being apart from dislocations.
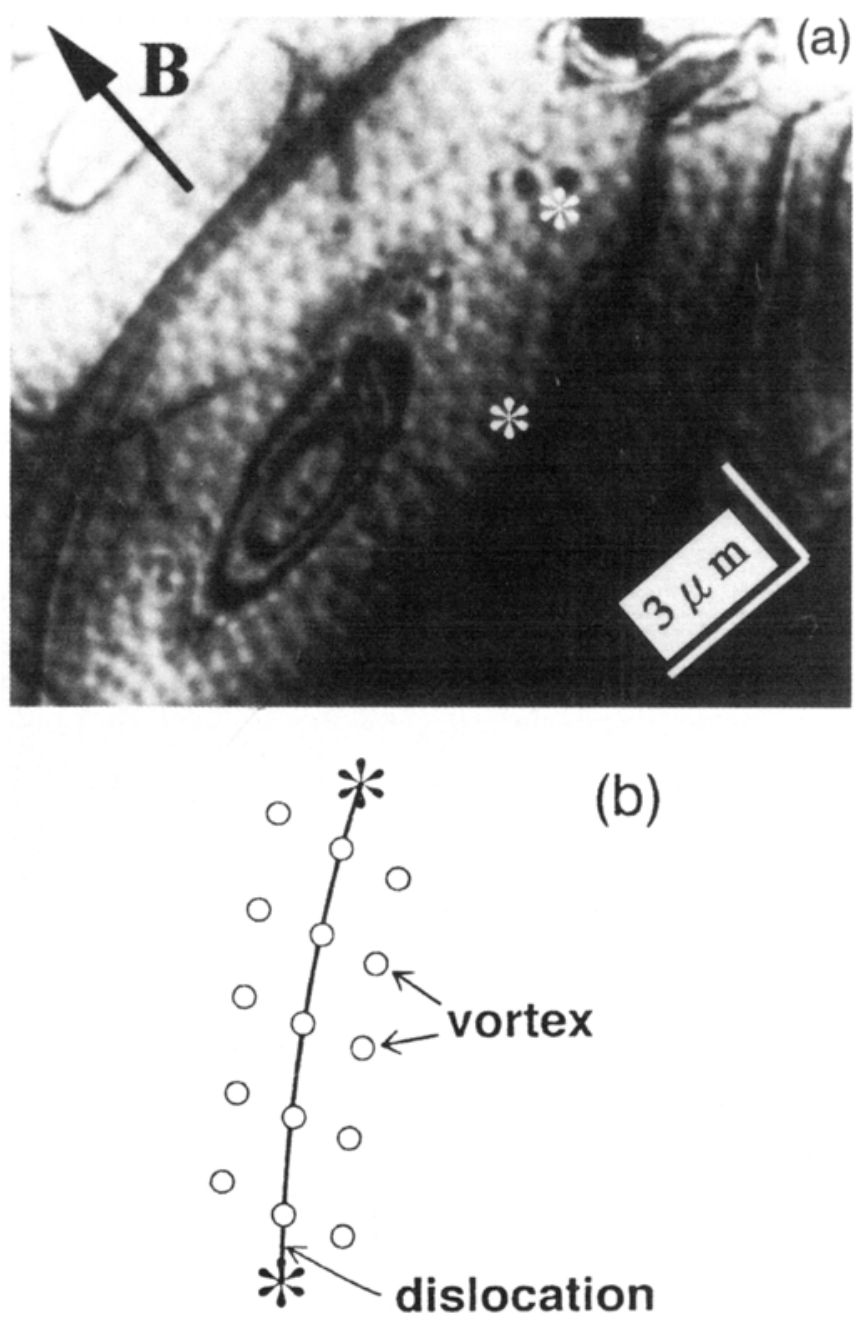

Figure 3. (a) A CLEM image showing the arrangement of vortices in a $\mathrm{Nb}$ film, which is cooled at $5 \mathrm{~K}$ under the magnetic field of $100 \mathrm{~K}$. The direction of magnetic field is shown by an arrow B. Thick lines are bend contours. A thin line, marked by asterisks, is a dislocation, near which vortices array in order. (b) A schematic representation of the regular array of vortices around a dislocation in (a). 

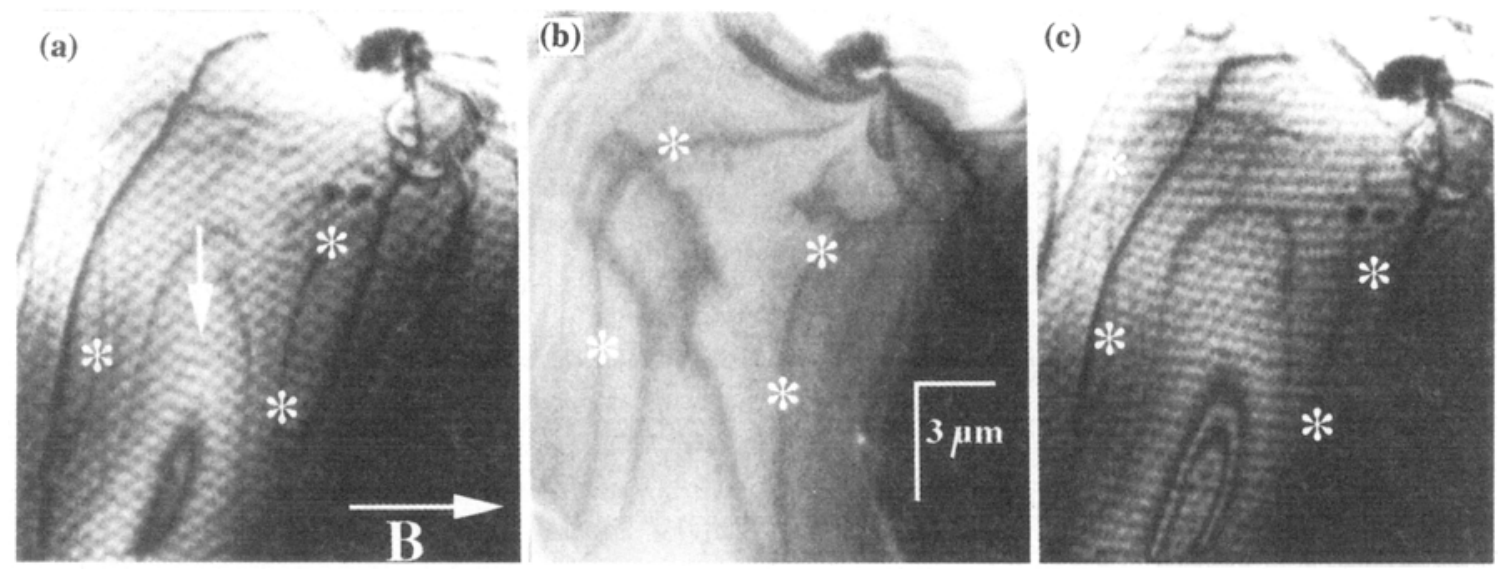

Figure 4. (a) A CLEM image showing a dense vortex lattice formed in a $\mathrm{Nb}$ film at $5 \mathrm{~K}$ under the magnetic field of $200 \mathrm{G}$. At the region, marked by a downward arrow, the vortex lattice is locally disordered. (b) and (c) CLEM images taken after increasing the specimen temperature to $20 \mathrm{~K}$ and decreasing back to $5 \mathrm{~K}$, respectively. The vortex lattice disappears in (b). In (c) the vortex lattice slightly changed the orientation as compared to (a).

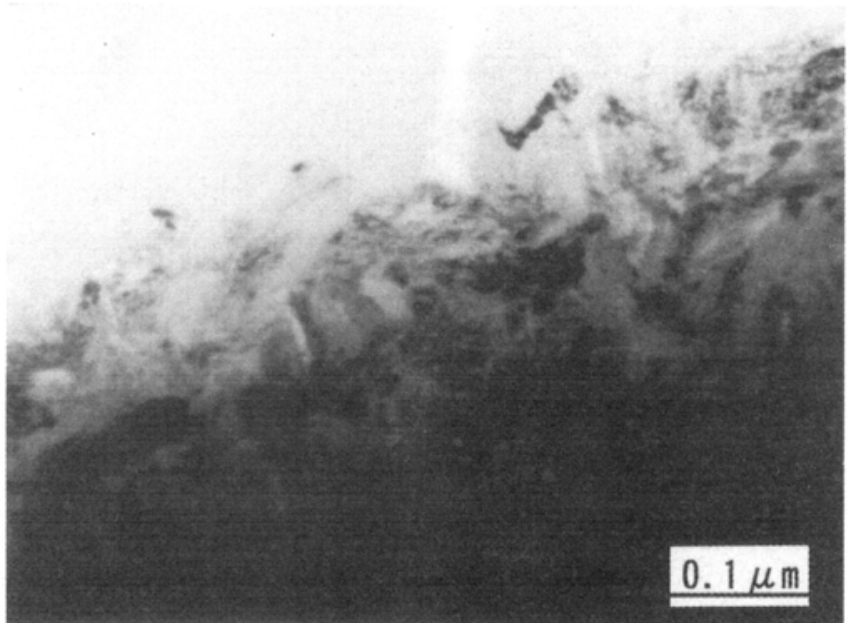

Figure 5. A bright field TEM image of NbTi. Small grains of $\mathrm{NbTi}$ are mixed with fine precipitates of $\alpha$-Ti.

The observations in figures $3 \mathrm{a}$ and $4 \mathrm{a}$ suggest that vortices are first pinned by dislocations and a vortex lattice is then formed around the pinned sites. Its extension and the density of vortices increase with the increase of magnetic field strength to form 'domains'. The disordered region in figure $4 \mathrm{a}$ must correspond to a boundary of domains. We may say that the preferential formation of vortex lattice near dislocations evidences the presence of the interaction between vortices and dislocations. It is interesting to note that similar domains have been observed also around artificial hole-like defects (Harada et al 1996).

Figure $4 \mathrm{~b}$ was taken from the same area as figure $4 \mathrm{a}$ after raising the temperature to $20 \mathrm{~K}$ and keeping the applied magnetic field at $200 \mathrm{G}$. It was noted that the vortex lattice had disappeared.

Figure $4 \mathrm{c}$ was taken after decreasing the specimen temperature back to $5 \mathrm{~K}$, under the magnetic field of $200 \mathrm{G}$. Vortices appeared again forming a dense lattice. The orientation of the lattice is slightly different from the previous one. Besides, the disordering in the lattice seems to be moderate. The lattice reorientation suggests that the vortex lattice is rather flexible depending on the mutual geometry between vortices pinned to a dislocation and surrounding unpinned ones and that the interaction between vortices and dislocations pinning them is rather weak.

Dislocations can be discriminated from bend contours by the observation as follows: on a slight change in the orientation of film, which is necessarily caused by the change in the specimen temperature, latter largely changes their sites, while the former little (compare figures $4 a$ with $\mathrm{b}$ and $\mathrm{c}$ !).

This observation strongly suggests that in-plane dislocations can act as rather strong pinning sites to cause the local ordering (figure 3a) and the domains of vortex lattice (figure $4 a$ ). It is clear that the in-plane dislocation can act as a pinning site for an array of vortices rather than a single vortex. This is the reason why in-plane dislocations can be more effective in the vortex pinning than edge-on dislocations.

\subsection{Observation of $\mathrm{NbTi}$}

We have tried to examine the mechanism of the vortex pinning in $\mathrm{NbTi}\left(T_{\mathrm{c}}=9.2 \mathrm{~K}\right)$ by applying the same method as above. Figure 5 is a bright-field TEM image of the 
specimen. Small grains of NbTi are mixed with fine precipitates of $\alpha$-Ti. But we have so far been unable to observe any clear image of vortices from this foil. The major reason for this may be the strong undulation on the foil surface. In order to overcome the problem we are now performing some trials to get specimens with smooth flat surface.

\section{Conclusion}

In low magnetic fields below $100 \mathrm{G}$ vortices with a low density are individually pinned to weak pinning site in a $\mathrm{Nb}$ foil. Their arrangement is irregular and variations in the density can be explained by the attraction of vortices to thinner areas due to the minimization of the line energy and the pinning mainly to edge-on dislocations. Thermal fluctuations can cause a single vortex to move between these weak pinning sites.

In magnetic fields around $100 \mathrm{G}$ the interaction of vortices with each other increases and their density becomes more uniform. The interaction of vortices with each other competes with the attractive force to individual pinning sites. They form locally a hexagonal arrangement. Around in-plane dislocations vortices start to array in order.

In magnetic fields of $200 \mathrm{G}$ the increased density leads to a strong vortex-vortex interaction and a dense, regular vortex lattice (Abrikosov lattice) is formed. Domains with slightly different orientations appear around dislocations with disordering in the regions in-between. The interaction of dislocations with vortices causes the regular arrangement of vortices around them. However, the interaction is not strong enough to form a unique arrangement; on increasing the temperature above $T_{\mathrm{c}}$ and lowering it again the vortex arrangement is similar but slightly reoriented. Besides, fast fluctuation in the vortex arrangement can be observed when the temperature is close to $T_{\mathrm{c}}$.

\section{Acknowledgements}

The authors are thankful to Hitachi Naka Works and Hitachi Advanced Research Laboratory for their collaboration on the development of the present CLEM, and to Drs M Nakajima and H Sakamoto, Furukawa Electric Co. Ltd. for providing the NbTi samples.

\section{References}

Cantoni M, Tanaka Y, Ishizuka M and Horiuchi S 1997 Physica C276 259

Cantoni M, Uchida M, Tsuruta T, Matsui $\mathrm{Y}$ and Horiuchi S 1998 J. Electr. Microsc. 47443

Harada K, Matsuda T, Bonevich J, Igarashi M, Kondo S, Pozzi G, Kawabe U and Tonomura A 1991 Nature 36051

Harada K, Matsuda T, Kasai H, Bonevich J E, Yoshida T, Kawabe U and Tonomura A 1993 Phys. Rev. Lett. 713371

Harada K, Kasai H, Matsuda T, Yamasaki M, Bonevich J E and Tonomura A 1994 Jpn J. Appl. Phys. 332534

Harada K, Kamimura O, Kasai H, Matsuda T, Tonomura A and Moshchalkov V V 1996 Science 2741167

Herring C P 1974 Phys. Rev. Lett. A47 105

Horiuchi S, Cantoni M, Uchida $\mathrm{M}$, Tsuruta $\mathrm{T}$ and Matsui $\mathrm{Y}$ 1998 Appl. Phys. Lett. 731293

Jeong D Y, He L L, Cantoni M and Horiuchi S 1996 Physica C269 279

Sow C H, Harada K, Tonomura A, Crastree G and Grier D G 1998 Phys. Rev. Lett. 802683

Wu X J, Ikeno Y, Kakimoto K and Horiuchi S 1991 Physica C174 423 